\title{
Uma Proposta para Avaliação da Virtualização de Funções de Rede em 5G
}

\author{
Cristoffer Leite $^{1}$, Priscila Solís Barreto ${ }^{1}$, Marcos F. Caetano ${ }^{1}$, \\ Eduardo Alchieri ${ }^{1}$ e Rafael Amaral ${ }^{1}$
}

\author{
${ }^{1}$ Departamento de Ciência da Computação - Universidade de Brasília (UnB) \\ Caixa Postal 4466 CEP 70910-900 - Brasília - DF - Brasil \\ cristoffer@aluno.unb.br, \{pris, mfcaetano, alchieri\}@unb.br
}

\begin{abstract}
In 5G, virtualization of network components is considered one of the key technologies that make up the evolution of architecture to provide flexible, sliced, and cost-effective services. Underlying hardware abstraction and its cost and portability advantages strongly motivate the use of virtualized functions, however, developing these environments for complex, multi-tiered architectures is still a challenging task that requires continuous work in defining standards and test platforms for validation. In this work, an experimental virtualized mobile network infrastructure that defines two evaluation functions in the $5 G$ architecture was designed, implemented and evaluated. The proposal differs from other studies by working with functions integrated with the $5 G$ architecture that can evaluate the control traffic of virtualized resources. The proposal was validated against a standard $5 G$ scenario and the results show that the approach resulted in a successful monitoring system with the potential to be integrated with the $5 G$ architecture as a support element for virtualized infrastructure optimization.
\end{abstract}

Resumo. No $5 G$, a virtualização de componentes de rede é considerada uma das principais tecnologias que compõem a evolução da arquitetura para prover serviços flexíveis, organizados em fatias e de baixo custo. A abstração do hardware subjacente e suas vantagens em termos de custo e portabilidade motivam fortemente o uso de funções virtualizadas. Entretanto, o desenvolvimento desses ambientes para arquiteturas complexas e de várias camadas ainda é uma tarefa desafiadora, que requer um trabalho contínuo na definição de padrões e plataformas de teste para validação. Neste trabalho foi projetada, implementada e avaliada uma infraestrutura experimental de rede móvel virtualizada que define duas funções de avaliação na arquitetura 5G. A proposta se diferencia de outros estudos ao trabalhar com funções integradas à arquitetura $5 G$ que podem avaliar o tráfego de controle dos recursos virtualizados. A proposta foi validada de forma bem sucedida em um cenário padrão do $5 G$, com resultados que permitem avaliar o comportamento do ambiente virtualizado e mostram o potencial da integração da proposta à arquitetura $5 G$ como elemento de suporte à otimização da infraestrutura virtualizada.

\section{Introdução}

A $5^{\text {a }}$ Geração de Comunicação Móvel (5G) concilia os sistemas de telecomunicações e a computação em nuvem através da Virtualização de Funções de Rede (Network Function 
Virtualisation, NFV). A Parceria Pública Privada 5G (5G Public Private Partnership, 5GPPP) introduz o conceito de NFV como uma tecnologia chave na definição da arquitetura do 5G [5GPPP 2017]. Essa inovação define a utilização de Função de Rede Virtualizada (Virtualised Network Function, VNF) equivalente no lugar de componentes físicos de rede. Cada VNF em uma rede virtualizada fornece serviços através de uma plataforma unificada, a adaptabilidade e a abstração de hardware subjacente motivam seu uso para reduzir custos de implementação e manutenção de infraestrutura [Basta et al. 2013].

A facilidade da infraestrutura com NFV também traz consigo alguns problemas típicos da virtualização, tais como a competição de recursos e degradação de desempenho [Herrera and Botero 2016], além de outros dilemas que exigem monitoramento constante dos recursos disponíveis. Na literatura, várias pesquisas utilizam sistemas de monitoramento distribuído e discutem os problemas relacionados à utilização de redes virtualizadas [Condoluci and Mahmoodi 2018, Laghrissi and Taleb 2019, Cao et al. 2016, Rosa et al. 2017]. Apesar disso, as abordagens dos trabalhos anteriores se concentram em analisar recursos de computação e memória, sem um foco específico na análise do tráfego gerado por essas implementações e seu impacto no desempenho.

Este trabalho apresenta uma solução para avaliação de funções de rede integradas à arquitetura NFV em redes 5G. O objetivo dessa integração é avaliar de forma precisa o impacto do tráfego produzido pela operação da infraestrutura virtualizada em cenários típicos de 5G. No melhor dos conhecimentos, este é o primeiro trabalho que propõe implementar funções de avaliação no Núcleo da Rede 5G (5G Core Network, 5GC) com foco na análise do tráfego de infraestrutura de rede.

O restante deste artigo está organizado da forma seguinte. A Seção 2 apresenta a revisão bibliográfica e os trabalhos relacionados. A Seção 3 apresenta a proposta e sua integração na arquitetura de NFV definida pelo Instituto Europeu de Padrões de Telecomunicações (European Telecommunications Standards Institute, ETSI) para o 5G, bem como as métricas definidas para avaliação. A Seção 4 detalha a implementação da proposta, além de apresentar o caso de uso escolhido para a avaliação em conjunto com as cargas de trabalho usadas nos testes e os resultados obtidos. Por fim, a Seção 5 apresenta a conclusão deste artigo e discute trabalhos futuros.

\section{Fundamentação Teórica e Trabalhos Relacionados}

\subsection{Padrão de NFV do ETSI}

O ETSI e um grupo de operadoras de redes móveis desenvolveram o padrão NFV, com o objetivo de transferir as funções de componentes intermediários, bem como seu gerenciamento, para aplicativos de software modularizados [ETSI 2013]. Uma vantagem do padrão proposto é que a mesma infraestrutura usada por um NFV pode ser usada para dar suporte a aplicativos de computação em nuvem simultaneamente, em um esquema de multilocação. O padrão NFV do ETSI implementa uma função de rede, anteriormente provida por um middlebox, por meio de uma entidade virtualizada chamada VNF. A VNF é executada na própria infraestrutura de computação em nuvem privada da operadora de rede móvel. Cada VNF implementa uma função distinta em uma Infraestrutura de Virtualização de Funções de Rede (Network Function Virtualisation Infrastructure, NFVI) e pode ser reproduzida ou migrada entre servidores em diferentes instâncias. A NFVI fornece computação e armazenamento ao mesmo tempo, de uma 
maneira muito similar ao conceito de Infraestrutura como Serviço (Infrastructure as a Service, IaaS) de uma Arquitetura Orientada a Serviços (Service-Oriented Architecture, SOA), mas também fornece recursos de rede de maneira semelhante a uma Rede como Serviço (Network as a Service, NaaS).

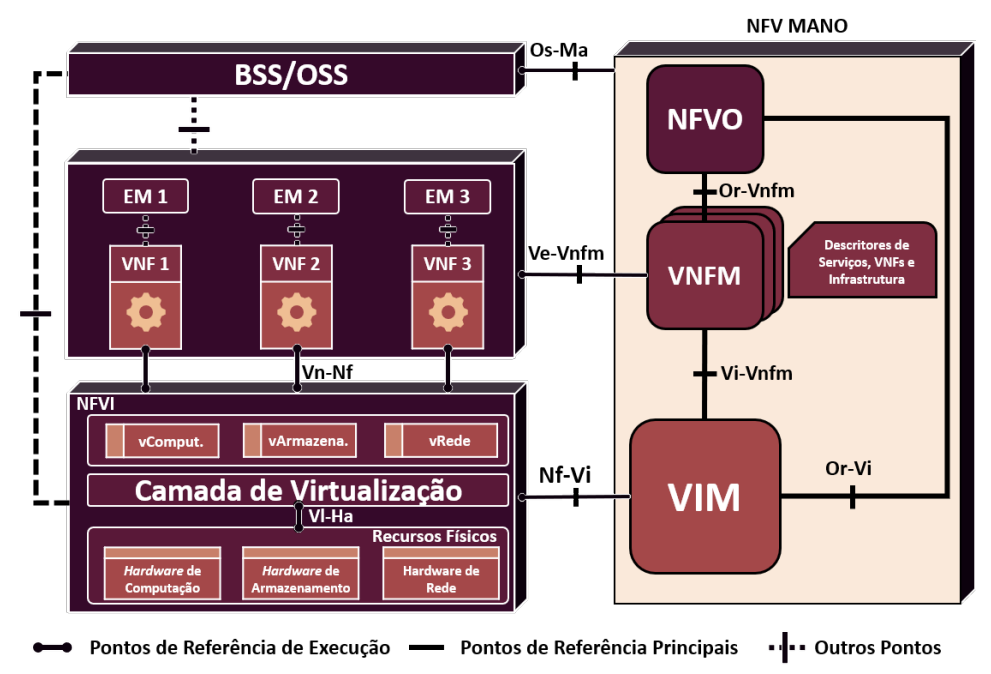

Figura 1. Arquitetura padrão ETSI NFV

A Figura 1 mostra a composição da arquitetura NFV do ETSI [ETSI 2014], com a camada Sistemas de Suporte a Operação e Negócios (Operation Support Systems /Business Support Systems, OSS/BSS), com a NFVI na parte inferior esquerda e o Gerenciador e Orquestrador (Management and Orchestration, MANO) de NFV à direita. O domínio do MANO proposto pelo ETSI abrange a orquestração e o gerenciamento do ciclo de vida e de todos os recursos físicos e de software, sendo responsável pelo controle da vida útil das VNFs e dividido em três componentes principais. O primeiro é chamado de Orquestrador de NFV (NFV Orchestrator, NFVO) e é responsável pelo gerenciamento de recursos de software e criação dos serviços na NFVI. Já o gerenciamento do ciclo de vida das VNFs reside no segundo componente do MANO, o Gerenciador de VNF (VNF Manager, VNFM). O último componente básico, o Gerenciador de Infrastrutura Virtualizada (Virtualised Infrastructure Manager, VIM), controla todas as interações entre o estrato superior do software e os recursos inferiores de hardware de computação, armazenamento e rede. Este último componente é responsável, entre outras funções, pela execução de regras de visibilidade de recursos e análise de desempenho da infraestrutura. Além disso, o MANO apresenta uma coleta de dados com cada Descritor de VNF (VNF Descriptor, VNFD) (um modelo de informações que define a operação de uma VNF), mantém um catálogo de recursos da NFVI, registra as instâncias VNF ativas e realiza a organização de cada Serviço de Rede (Network Service, NS) implantado.

\subsection{Componentes de Núcleo da Rede 5G e Arquitetura Orientada a Serviços}

A rede $5 \mathrm{G}$ usa um componente de rede principal chamado de 5GC, com alterações significativas sobre a arquitetura Evolved Packet Core (EPC) do Long-Term Evolution (LTE). O novo núcleo apresenta uma Arquitetura Baseada em Serviços (Service-Based Architecture, SBA) como um sistema de comunicação alternativo entre o núcleo de Função de Rede (Network Function, NF) em 5G. 


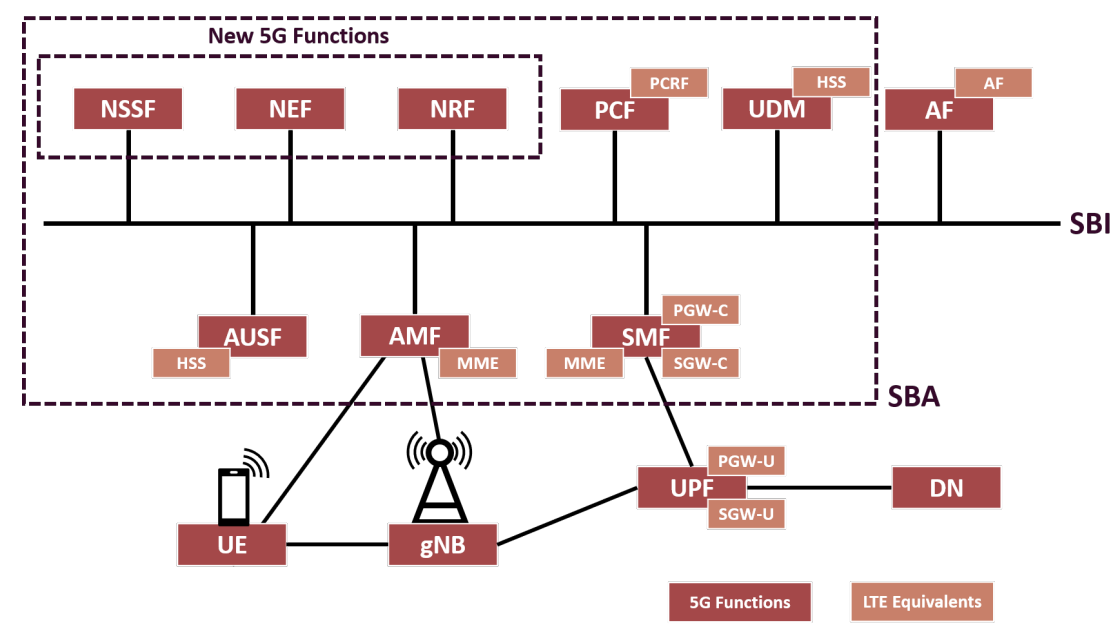

Figura 2. Núcleo da Rede 5G e seus equivalentes LTE

A Figura 2 mostra o grupo NF do 5GC SBA, a Interface Baseada em Serviços (Service-Based Interface, SBI) e ilustra as suas contrapartes LTE para uma melhor compreensão. No núcleo tem destaque as funções de Função de Gerenciamento de Acesso e Mobilidade (Access and Mobility Management function, AMF), que é responsável pelo gerenciamento das atribuições de mobilidade e a supervisão do controle de acesso. A AMF interage com muitas outras NF para executar tarefas cooperativas, como autenticação na Função de Autenticação de Servidor (Authentication Server Function, AUSF), controle de assinatura na Gerenciamento Unificado de Dados (Unified Data Management, UDM), orquestração de fatias na Função de Seleção de Fatia (Network Slice Selection Function, NSSF) e manipulação de Unidade de Dado de Protocolo (Protocol Data Unit, PDU). A Função de Gerenciamento de Sessão (Session Management Function, SMF) gerencia funções de Entidade de Gerenciamento de Mobilidade (Mobility Management Entity, MME), que tratam de governança da sessão (gerenciamento, estabelecimento, modificação e liberação). O bloco Função do Plano do Usuário (User Plane Function, UPF) executa as funções do plano de usuário além de integrar políticas de Qualidade de Serviço (Quality of Service, QoS), restrição, redirecionamento e direcionamento de tráfego, a partir do bloco Função de Politicas e Carregamento (Policy and Charging Rules Function, PCRF). Sendo também responsável pela inspeção, roteamento e encaminhamento de pacotes e é o ponto de sessão externo do PDU para qualquer rede de dados (Data Network (DN)). O Equipamento do Usuário (User Equipment, UE) representa qualquer equipamento conectado à rede $5 \mathrm{G}$ e o Nó $\mathrm{B}$ de Nova Geração (Next Generation $N o d e B, \mathrm{gNB}$ ) é o nó de comunicação ou estação rádio base. Um detalhamento mais aprofundado da arquitetura pode ser consultado em [5GPPP 2017].

Conforme o relatório 3GPP TS 23.501 [3GPP 2018], os serviços no 5G interagem por interfaces de microsserviço padronizadas em uma arquitetura modular de ProvedorConsumidor. Cada microsserviço possui a definição de um procedimento que permite a comunicação direta entre diferentes funções usando uma SBI unificada. Toda a arquitetura segue implementações de recursos sem estado, dissociando recursos de computação de recursos de armazenamento. A Figura 3 mostra uma implementação SBA a partir da interação entre uma função de rede consumidora e provedora. A comunicação pode seguir tanto uma interação Inscrição-Notificação quanto Requisição-Resposta através de 
chamadas HTTP simples.
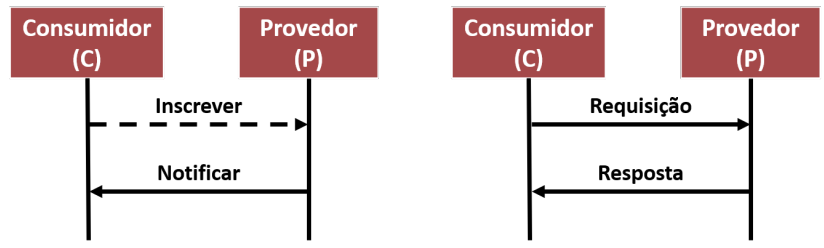

Figura 3. Interações entre uma NF Consumidora e uma NF Provedora

\subsection{Implementação do $5 \mathrm{G}$ em NFV}

A arquitetura do 5G pode ser implementada como uma composição de recursos físicos e virtuais. A Figura 4 mostra um exemplo de um ambiente 5G com NFV e NFVI conforme os padrões do ETSI. À esquerda, o ambiente possui um grupo de funções físicas e virtualizadas. À direita, um exemplo de como um NFVI dá suporte para essa arquitetura. No caso mostrado, há dois nós de computação, quatro máquinas virtuais e um grupo de recursos virtualizados sendo utilizados para dar suporte à implementação das VNFs. Além disso, os comutadores virtuais (vSwitch) criados pelo VIM geram uma rede virtual para endereçar todos os pacotes transferidos entre recursos virtuais.

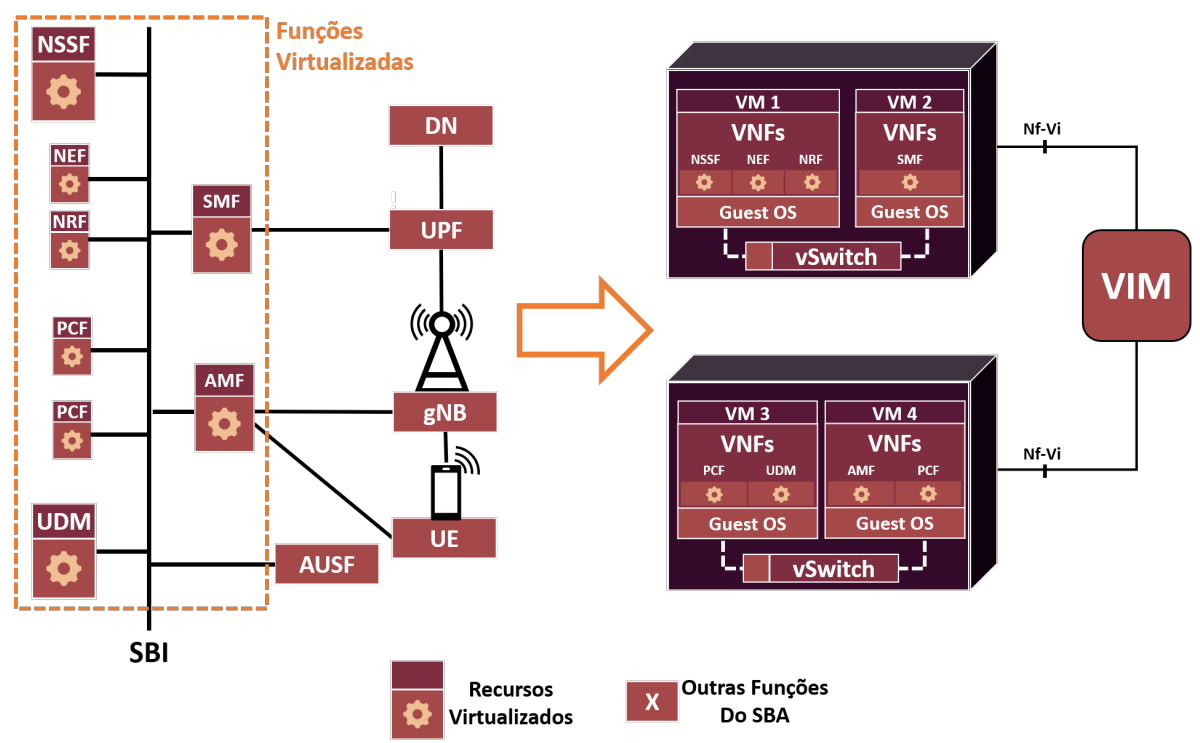

Figura 4. Exemplo de Implementação de 5G em uma Infraestrutura Virtualizada

O uso de NFV permite a migração de funções pela rede através da abstração da infraestrutura. O NFV no 5G propõe a abstração da infraestrutura de recursos para permitir o uso de elementos lógicos de rede na implementação e implantar funções de rede em dispositivos de borda. A computação de borda apresenta desafios relacionados à colocação de funções de rede e a como distribui-las para evitar impactos na QoS percebida pelos usuários [Taleb et al. 2015]. Sobre agendamento de NFV, o trabalho de [Riera et al. 2014] introduziu uma definição formal do problema que representa a dificuldade de escolher entre os recursos disponíveis para o instanciamento de uma VNF e mostrou que o problema em questão é equivalente ao Problema de Agendamento de Projetos com Restrição de Recursos (Resource-Constrained Project Scheduling Problem, RCPSP), um problema de classe NP-Difícil. 


\subsection{Trabalhos Relacionados}

De acordo com [Condoluci and Mahmoodi 2018], as abordagens mais recentes de análise de desempenho de NFV concentram-se apenas na investigação da integração vertical e posicionamento de funções de rede [Basta et al. 2013, Taleb et al. 2015]. Outros trabalhos tentam reduzir o impacto do posicionamento ao prever requisitos de VNFs. O estudo de [Laghrissi and Taleb 2019] divide os trabalhos nessa área por posicionamento de funções gerais e posicionamento de funções específicas: o primeiro trata de estratégias de posicionamento, como replicação, gráfico de encaminhamento e política de encadeamento, enquanto que o segundo se concentra apenas em serviços específicos, geralmente em $g a$ teways, mas também em cache e inspeção de pacotes virtuais.

Em [Cao et al. 2016] foi desenvolvida uma estrutura de caracterização de desempenho para VNF em nuvem privada. A estrutura introduzida concentra-se na estimativa da capacidade da VNF para a composição dos NS e na análise de VNF em cluster para detecção de gargalos. Um fator essencial da pesquisa acima é o uso de Clearwater, um Subsistema Multimídia IP (IP Multimedia Subsystem, IMS) com uma implantação típica VNF para teste. O cenário pretendido analisa um componente de rede móvel virtualizado usando um ambiente Session Initiation Protocol (SIP). A estrutura mostra um sucesso considerável ao analisar a utilização da CPU e o dimensionamento de componentes, mesmo com diferentes padrões de uso.

O estudo de [Rosa et al. 2015] apresenta uma estrutura para a construção de perfil de desempenho de VNF. Este serviço analisa o impacto de cada VNF e cria um histórico de benchmarking. Esse trabalho foi aprimorado posteriormente em [Rosa et al. 2017], com uma nova estrutura chamada GYM, que define interfaces padronizadas para a análise VNF e permite entradas de teste definidas pelo usuário. A estrutura proposta tenta vincular os resultados de algumas ferramentas de análise de tráfego e visa criar uma metodologia reutilizável que possa ser integrada aos orquestradores NFV para facilitar o processo e evitar o excesso de capacidade. O trabalho de [Nam et al. 2018] avalia o desempenho de várias VNFs ao longo de uma cadeia de serviços de rede para detectar operações irregulares. A ferramenta cria autonomamente perfis com problemas de desempenho encontrados em diferentes VNFs, ao analisar a relação entre o hypervisor e as funções em cada camada do ambiente NFV implementado.

Dos trabalhos relacionados se conclui que, até a presente data, não há nenhum trabalho que analise a eficiência do tráfego de infraestrutura de virtualização integrada à arquitetura 5G. Assim, o objetivo deste trabalho é propor um sistema automatizado de avaliação de redes virtualizadas com base na porcentagem de tráfego adicional gerado pela infraestrutura ao manter a NFV ativa. O modelo de avaliação proposto tem a intenção de adicionar esse serviço como um componente nativo ao 5GC, para monitorar em tempo real o ambiente virtualizado.

\section{Proposta do Modelo de Avaliação de Virtualização de Funções em 5G}

Nesta seção serão detalhadas as duas novas funções propostas que serão integradas à arquitetura 5G SBA e as métricas definidas para avaliação.

\subsection{Funções de Observação do Ambiente Virtualizado}

A solução propõe o monitoramento do SBI em cada nó de computação usando uma nova Função de Observação (Observation Function, ObF), que encaminha os dados coletados 
para uma nova Função de Avaliação de Performance (Performance Evaluation Function, PEvF) centralizada, responsável por monitorar todos os recursos virtuais e gerar análises consolidadas. A Figura 5 mostra a integração das novas funções no $5 \mathrm{GC}$ utilizando a comunicação simples do SBI como ponto de acesso.

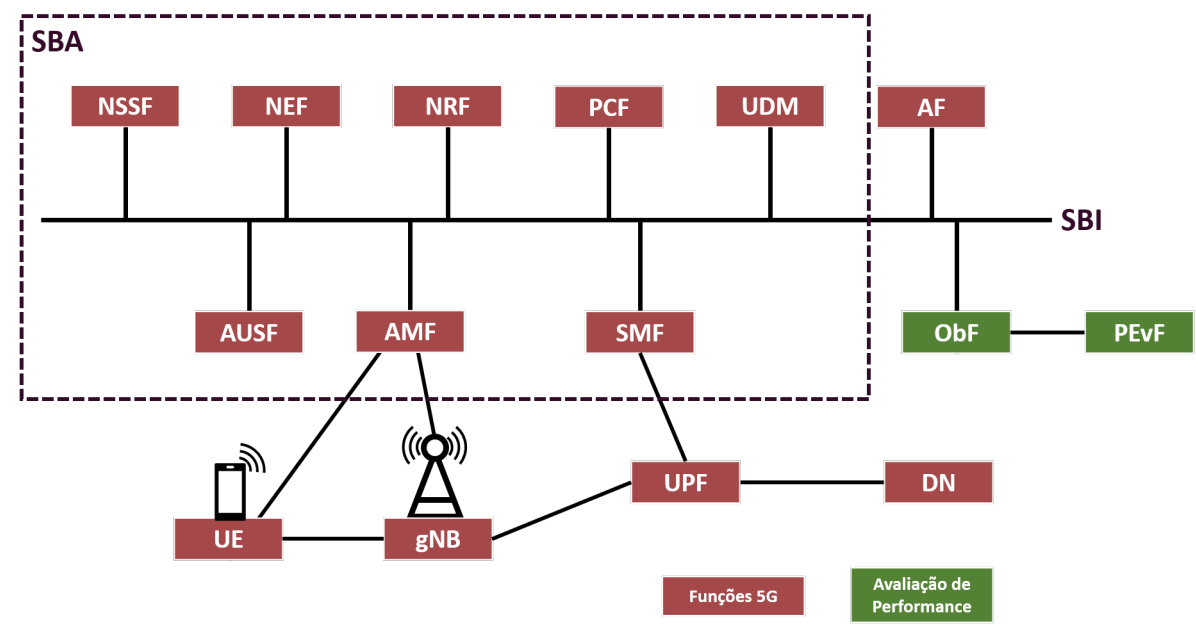

Figura 5. 5GC com as Funções Propostas

Para executar o serviço, uma ObF local escutará o vSwitch principal em todos os nós de computação, monitorando os dados transferidos e realizando a inspeção de pacotes. Essa ação fornecerá ao PEvF os dados necessários para gerar uma avaliação. A Figura 6 mostra como habilitar a adição desses componentes em um NFVI usando uma configuração simples. Para evitar mais competição de recursos com outros componentes virtualizados, a ObF será uma implementação não virtualizada. Essa opção também simplifica o desenvolvimento e cria um componente sólido com pouco impacto na arquitetura geral. A PEvF monitora continuamente as interfaces do VIM e do VNFM, além de também agregar várias métricas coletadas pelas ObFs distribuídas. No VIM, ela recebe informações para criar um mapa de infraestrutura virtualizada e um mapa de fatias do NFV. Das ObFs, ela recebe informações coletadas dos nós de computação. A PEvF consolida todas as informações coletadas e, em seguida, usa os mapas gerados para criar eventos e notificações sobre a integridade da virtualização.

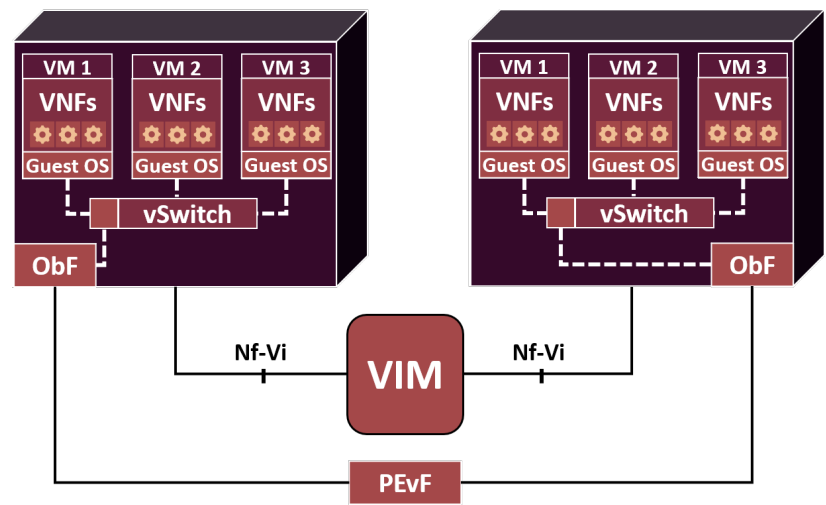

Figura 6. Habilitação dos Novos Componentes do NFVI Para Avaliação 


\subsection{Métricas de Avaliação}

O relatório ETSI GS NFV-TST 008 V2.4.1 [ETSI 2018] descreve e analisa um grupo de métricas para medir a QoS percebida pelo consumidor de qualquer NFV em relação aos recursos físicos e seus equivalentes virtuais. A avaliação de desempenho deste trabalho visa verificar a eficiência do encaminhamento de dados, analisando a quantidade de dados úteis que flui dentro da rede. A Tabela 1 mostra as métricas que serão usadas pela PEvF para avaliação de desempenho, as quais fornecem informações únicas que agregam valor primordial à avaliação [Jain 1991]. A Contagem de Pacotes (N.1) representa o número de pacotes transferidos com êxito por uma interface, a Contagem de Octetos (N.2) representa a quantidade total de bytes transferidos por uma interface, considerando a soma de todos os pacotes entregues com sucesso, e Contagem de Pacotes Descartados (N.3) representa o número de transmissões malsucedidas de pacotes que ocorreram devido à falta de recursos. Para avaliar a eficiência no ambiente virtualizado, as métricas anteriores serão comparadas com a Quantidade de Dados Úteis (N.4), que representam os dados de usuários efetivamente transmitidos e a Quantidade de Dados de Infraestrutura (N.5).

Tabela 1. Métricas de Análise

\begin{tabular}{|l|l|l|l|l|}
\hline Recurso & Código & Métrica & Unidade & Fonte \\
\hline \multirow{3}{*}{ Rede } & N.1 & Contagem de Pacotes & Número (\#) & ObF \\
\cline { 2 - 5 } & N.2 & Contagem de Octetos & Número (\#) & ObF \\
\cline { 2 - 5 } & N.3 & Contagem de Pacotes Descartados & Número(\#) & ObF \\
\cline { 2 - 5 } & N.4 & Dados Úteis & Porcentagem (\%) & ObF \\
\cline { 2 - 5 } & N.5 & Dados de Infraestrutura & Porcentagem (\%) & ObF \\
\hline
\end{tabular}

\section{Implementação e Resultados Experimentais}

\subsection{Implementação da Arquitetura Experimental de NFV 5G}

$\mathrm{Na}$ implementação da arquitetura experimental foi utilizado o Open Source MANO (OSM) Versão 5 [ETSI 2019a] como NFVO e VNFM, enquanto o OpenStack (Ocata) [Sefraoui et al. 2012] operou como VIM. Essas opções seguem o padrão utilizado de fato por muitos projetos 5GPPP [Rebecchi et al. 2019, Rizou et al. 2018, Khalili et al. 2019]. No VNFM, o OSM fornece instanciação de VNF nativa, inicialização de serviço e gerenciamento de tempo além de execução dos serviços virtualizados, o que permite um gerenciamento completo do ciclo de vida. Para NFVO, o OSM também fornece gerenciamento de recursos de software [ETSI 2019b]. O Openstack permite a implementação de recursos de computação, armazenamento (ambos através do componente Nova) e de rede (através do componente Neutron) em uma NFVI para toda a arquitetura. Essa combinação permite seguir as diretrizes ETSI para ambientes de rede virtualizados.

A infraestrutura física de testes foi definida com um servidor em uma unidade centralizada que age como um NFV MANO, e o NFVI, este último composto por um grupo de nós de computação atuando como unidades distribuídas que disponibilizam recursos virtuais para a implantação de VNFs. O servidor é um Mini-ITX i5 3Ghz 8 GB de memória e 1 TB de disco, operando com o Ubuntu Server 18.04 para dar suporte ao Hyper-V. Em uma Máquina Virtual (Virtual Machine, VM) é executado o controlador OpenStack Ocata, juntamente com a versão cinco do OSM. Essa VM possui três interfaces de rede. A primeira fornece comunicação do VIM entre o controlador OpenStack 
e o NFVI. A segunda interface é para o gerenciamento do ciclo de vida de VNF pelo OSM. E a última interface fornece conectividade e permite a configuração remota do servidor. Os nós de computação são um grupo de diferentes versões Raspberry Pi (RPi) (3B, 3B +, 4 e Zero W), que emulam um cenário de teste de baixo custo e com restrição de energia. Todos os RPi funcionam com o Raspbian Release 06-2019 e executam os componentes Openstack Nova e Neutron. Todos os RPis possuem as três interfaces iguais às do servidor.

\subsection{Definição do Caso de Uso e Carga de Trabalho Utilizada}

A 5GPPP define uma gama de possíveis aplicações nas redes $5 \mathrm{G}$, agrupadas em três casos de uso [Popovski et al. 2018]: Banda Larga Móvel Aprimorada (Enhanced Mobile Broadband, eMBB), Comunicação Massiva de Tipo de Máquina (Massive Machine Type Communication, mMTC) e Comunicação Ultra-Confiável e de Baixa Latência (UltraReliable and Low Latency Communications, URLLC). Cada tipo deve atender a diferentes métricas de QoS [ITU Recommendation G.1010 2001]. Os casos de uso do eMBB exigem altas taxas de download, alta densidade de conectividade, ampla mobilidade e cobertura. URLLC exige uma combinação de baixa latência e alta disponibilidade para atingir aproximadamente $99,9 \%$ de confiabilidade e melhorar os cenários de computação de borda. O mMTC exige alta densidade de tráfego e interconexão de muitas tecnologias diferentes.

No experimento, o caso de uso a ser avaliado é o mMTC, caracterizado por testes de conexões de dispositivos da Internet das Coisas (Internet of Things, IoT) temporizadas [3GPP 2014]. Em ambientes IoT com um grande número de dispositivos que enviam dados para uma nuvem, podem ser implementados pontos intermediários para balanceamento de carga, em que o HAProxy é considerado uma implementação apropriada [Hou et al. 2016]. Por esse motivo, os testes no cenário mMTC usam a implementação virtual descrita na Figura 7a, com o NFV MANO na função de balanceador de carga, que envia dados para o componente virtual no Ubuntu Xenial, conforme ilustrado na Figura $7 \mathrm{~b}$.

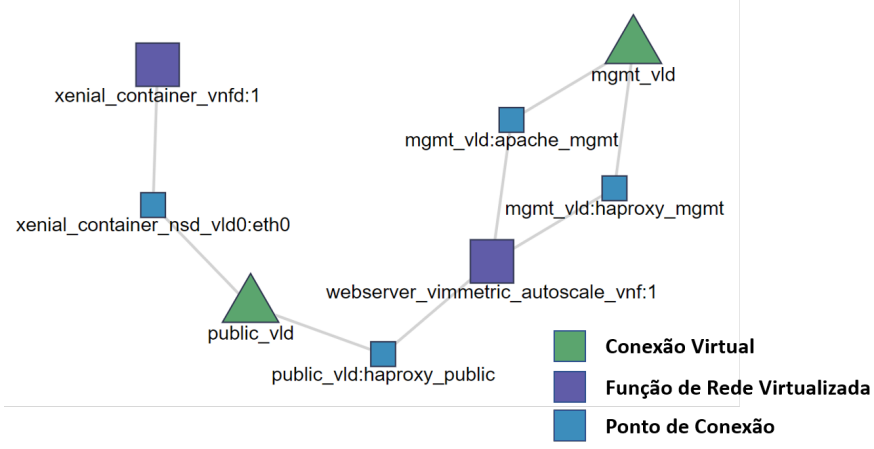

(a) Componentes Virtuais e Ligações Virtuais

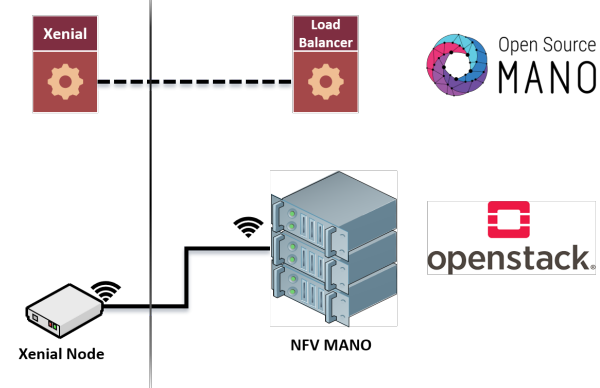

(b) Infraestrutura Física e Virtualizada

Figura 7. Implementação do Cenário de Testes para o mMTC

No ambiente de teste, foram emuladas fontes homogêneas de pacotes modeladas com base em aplicações mMTC em que o tráfego flui em pequenas mensagens de comunicação temporizadas [Laner et al. 2014], enviadas por uma grande 
quantidade de dispositivos. Trabalhos relacionados identificam o processo de Poisson [3GPP 2014] como um modelo adequado para simular o padrão de comunicação mMTC, representado por uma combinação de muitos dispositivos IoT temporizados [Hossfeld Tobias et al. 2018] em ambiente de grande escala. No experimento foi gerada uma carga de trabalho sintética que segue essas especificações.

Além disso, com base no relatório [3GPP 2011], um cenário urbano possui uma densidade aproximada de 4.968 dispositivos de IoT por célula. Ao considerar os requisitos mínimos para o tráfego mMTC [Itu-r 2017], para fornecer uma qualidade viável de downlink para comunicação de máquina, um pacote médio deve possuir 252 bytes. De acordo com [Sivanathan et al. 2017], ao considerar o tempo médio de atividade de 8 segundos e o tempo de inatividade de 241 segundos para dispositivos com tamanho aproximado de pacote de 234 bytes, cada dispositivo produz em média 145 pacotes durante o período mais ativo do dia, que é de 10 horas. Nos testes foi definido um tempo de espera de 1 segundo entre cada transmissão com base em uma distribuição uniforme. Dessa forma, a janela de transmissão necessária para a mesma quantidade de tráfego é de 135 segundos. Com base nesses critérios, a carga de trabalho utilizada no experimento constou de 127 amostras de 135 segundos, coleta que representa aproximadamente 1 hora de dados para as informações agregadas de 4.968 dispositivos de IoT homogêneos.

\subsection{Resultados e Análises}

A Figura 8 mostra um exemplo de uma das janelas de monitoramento de 135 segundos usadas no experimento para um cenário mMTC, conforme a carga de trabalho definida na seção anterior. No gráfico é observada uma flutuação de dados na conexão para a infraestrutura e o tráfego Comunicação Tipo Máquina (Machine Type Communication, MTC) no ambiente virtualizado. Pode ser observado que o tráfego MTC é equivalente aos dados enviados pelo Load Balancer VNF no NFV MANO para o Xenial Node VNF em execução no nó de computação remoto, o que mostra a validação do funcionamento do ambiente de teste. Conforme definido na Seção 3, um ObF distribuído monitora esses dados e relata todas as informações ao PEvF centralizado. Pode ser observado que a verificação de integridade do OpenStack acontece a cada 60 segundos, os dados da infraestrutura respondem por todo o tráfego gerado pelas mensagens enviadas pelo nó de computação e também pelo servidor centralizado.

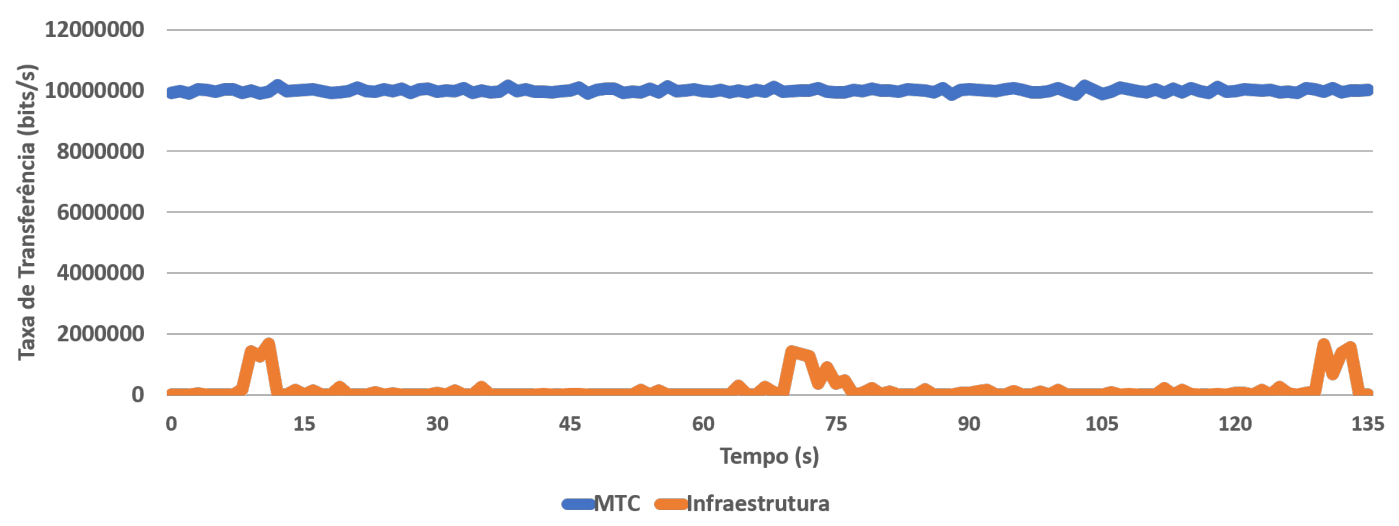

Figura 8. Comparação da Taxa de Transferência Durante uma Transmissão de Carga de Trabalho para mMTC 
$\mathrm{Na}$ amostra específica apresentada na Figura 8, o tráfego do tipo máquina segue um ritmo constante com pequenas variações na taxa de transferência. A transmissão estável ocorre devido às características temporizadas desses dispositivos [3GPP 2014]. Com uma taxa de transferência média de 10,07 Mbps, a amostra gerou um total de 683899 pacotes (N.1) nessa transmissão única, fornecendo uma média de 5069,92 pacotes por segundo, que produziu 169985155 bytes (N.2) de acordo com a detecção ObF, e ainda teve 6599 pacotes descartados (N.3) ou 0,965 \%. A Figura 9 mostra o número de pacotes transferidos durante essa amostra de teste específica. O número de pacotes se assemelha às variações da taxa de transferência porque o tráfego do tipo máquina geralmente usa tamanhos padrão para mensagens trocadas entre entidades [Ito et al. 2014]. Dada a distribuição Poisson do tráfego mMTC para comunicação temporizada IoT, o número de pacotes geralmente se aproxima do número de dispositivos que enviam informações a qualquer momento.

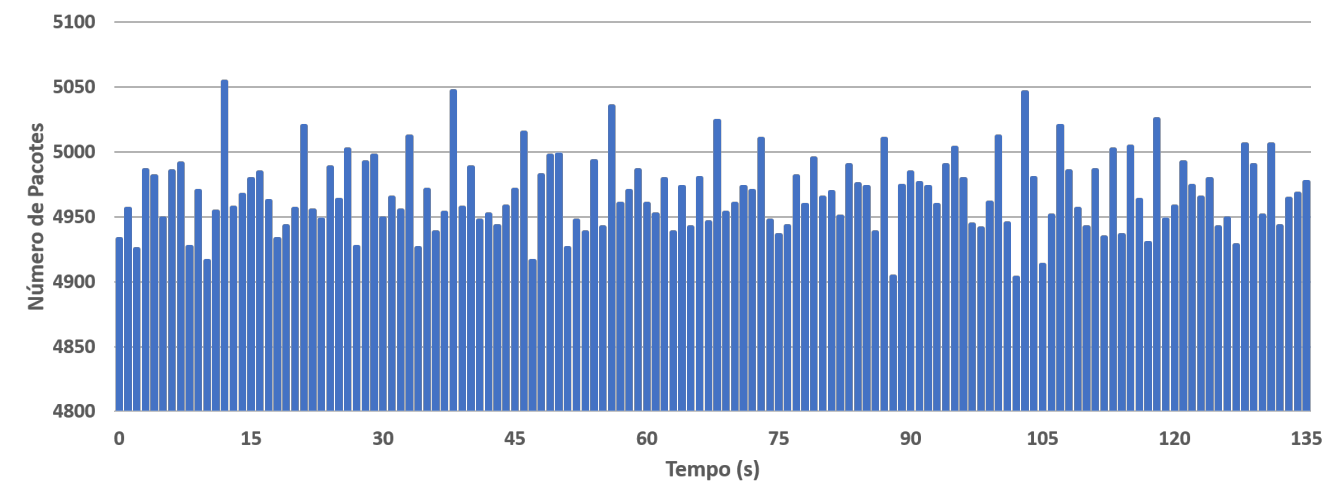
Figura 9. Número de Pacotes Transferidos para uma Amostra mMTC de 135 Se-
gundos

As ObFs coletaram aproximadamente 1 hora de carga de trabalho sintética mMTC e mensagens de controle de infraestrutura. Durante as rotinas de teste completas, o PEvF consolidou informações para calcular uma taxa de transferência média de 9,98 Mbps, com uma população total de 27 testes de 135 segundos e um desvio padrão de 2,9767, com um intervalo de confiança de $95 \%$. Durante uma execução média de 135 segundos, os dados úteis corresponderam a cerca de 169,98 MB, enquanto a infraestrutura produziu 2,69 MB, o que equivale a apenas 1,55 \% (N.5) do total de informações transmitidas no ambiente.

Como mostra a Figura 10, nas verificações de integridade, o rendimento da infraestrutura ainda aumenta drasticamente. Ao separar a comunicação entre períodos normais e períodos de verificação de integridade, a infraestrutura corresponde a uma média de $0,52 \%$ do tráfego total no primeiro caso e 11,16 \% no segundo caso. Mesmo em períodos de verificação de integridade, o mMTC mantém uma taxa estável, o que sugere que a operação do NFV não provoca aumento intenso do tráfego. A Tabela 2 apresenta as métricas finais coletadas para este caso de uso.

Os resultados obtidos demonstram que a proposta funciona corretamente na infraestrutura distribuída e permite a geração de informações adequadas sobre o comportamento do ambiente virtualizado. A proposta é uma abordagem inovadora para coleta de dados em ambientes NFV e os resultados obtidos motivam o uso das duas funções como parte integrante do 5G SBA para a avaliação de infraestruturas orientadas a recursos. 


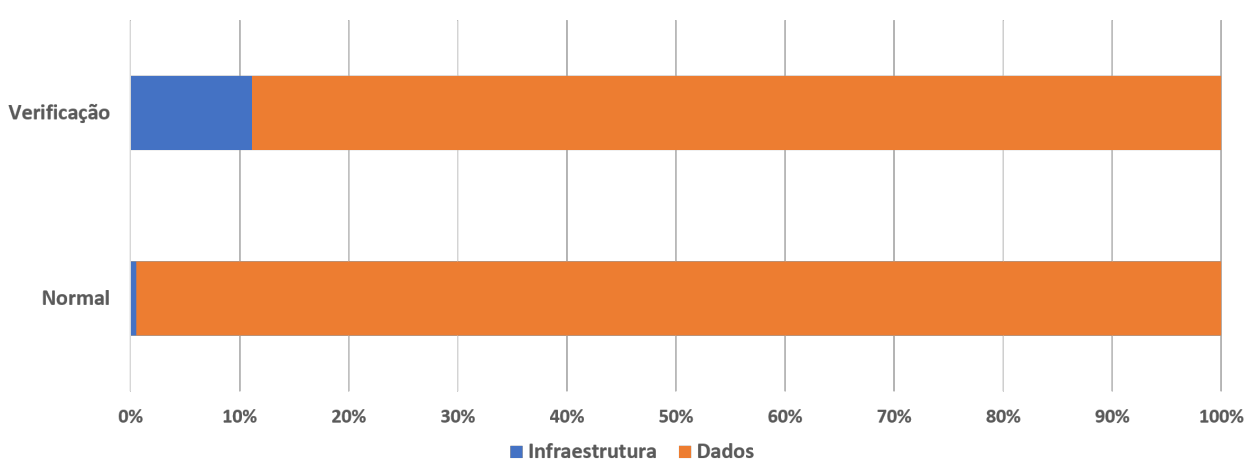

Figura 10. Diferença entre a Quantidade de Dados Transferidos nos Períodos Normais e de Verificação de Saúde para um Cenário do mMTC

Tabela 2. Resultados da Avaliação de Eficiência para uma Transmissão mMTC de 135 Seconds

\begin{tabular}{|l|l|l|l|}
\hline Recurso & Código & Métrica & Unidade \\
\hline \multirow{4}{*}{ Rede } & N.1 & Contagem de Pacotes & $683899(\#)$ \\
\cline { 2 - 4 } & N.2 & Contagem de Octetos & $169985155(\#)$ \\
\cline { 2 - 4 } & N.3 & Contagem de Pacotes Descartados & $6599(\#)$ \\
\cline { 2 - 4 } & N.4 & Dados Úteis & $98.45(\%)$ \\
\cline { 2 - 4 } & N.5 & Dados de Infraestrutura & $1.55(\%)$ \\
\hline
\end{tabular}

\section{Conclusões e Trabalhos Futuros}

Neste trabalho foi projetada, implementada e avaliada uma infraestrutura experimental de rede móvel 5G virtualizada que define uma função de avaliação integrada ao SBA 5G. A proposta se diferencia de outros trabalhos, que propõem criar um sistema de monitoramento de desempenho para NFV, mas que não abordam a integração nativa no 5G SBA para avaliar o desempenho de redes móveis virtualizadas com foco no tráfego adicional produzido pela própria infraestrutura. A proposta apresenta duas novas funções no SBA, uma Função de Observação (ObF) distribuída e uma Função de Avaliação de Desempenho (PEvF) centralizada. A proposta foi validada em um cenário configurado como mMTC com cargas de trabalho típicas, usando as funções de observação anexadas a comutadores virtuais em nós de computação distribuídos. Os resultados mostram que no cenário de avaliação, a porcentagem de tráfego adicional criado pela infraestrutura diminui quando aumenta a quantidade de dados úteis.

Trabalhos futuros devem considerar o uso de uma maior quantidade de nós de computação para melhor consolidar a avaliação e considerar os resultados como elementos para otimizar o processo de verificação do OpenStack Nova nos recursos de rede.

\section{Agradecimentos}

Este trabalho teve o apoio do projeto 5G-Range (www.5g-range.eu), aprovado na 4a. Chamada EU-BR em TICs, financiada pelo CTIC/RNP/MCTIC e do Edital 04/DPI/2019 de Apoio a Pesquisadores da Universidade de Brasília. 


\section{Referências}

3GPP (2011). 3GPP TR 37.868 V11.0.0 - Study on RAN Improvements for Machine-type Communications. 3GPP TR 37.868 V11.0.0.

3GPP (2014). 3GPP TR 43.868 V12.1.0 - GERAN improvements for Machine-Type Communications (MTC). 3GPP TR 43.868 V12.1.0 Rel. 12.

3GPP (2018). ETSI TS 123501 - V15.3.0 - 5G; System Architecture for the 5G System. ETSI TS 23.501 V15.3.0 Rel. 15, 0.

5GPPP (2017). View on 5G Architecture (Version 2 . 0). (July).

Basta, A., Kellerer, W., Hoffmann, M., Hoffmann, K., and Schmidt, E. D. (2013). A virtual SDN-enabled LTE EPC architecture: A case study for S-/P-gateways functions. Technical report.

Cao, L., Sharma, P., Fahmy, S., and Saxena, V. (2016). NFV-VITAL: A framework for characterizing the performance of virtual network functions. 2015 IEEE Conference on Network Function Virtualization and Software Defined Network, NFV-SDN 2015, pages 93-99.

Condoluci, M. and Mahmoodi, T. (2018). Softwarization and virtualization in 5G mobile networks: Benefits, trends and challenges. Computer Networks, 146:65-84.

ETSI (2013). GS NFV 001 V1.1.1 - NFV: Use Cases Group Specification. GS NFV 001 - V1.1.1.

ETSI (2014). GS NFV 002 V1.2.1 - NFV: Architectural Framework. GS NFV 002 V1.2.1, 1:1-21.

ETSI (2018). ETSI GS NFV-TST 008 V2.4.1 - Network Functions Virtualisation (NFV) Release 2; Testing; NFVI Compute and Network Metrics Specification. ETSI GS NFVTST 008 V2.4.1.

ETSI (2019a). OSM Release FIVE - Technical Overview. OSM White Paper.

ETSI (2019b). OSM White Paper - OSM VNF Onboarding Guidelines. OSM White Paper.

Herrera, J. G. and Botero, J. F. (2016). Resource Allocation in NFV: A Comprehensive Survey. IEEE TRANSACTIONS ON NETWORK AND SERVICE MANAGEMENT, 13(3).

Hossfeld Tobias, Metzger, F., and Heegaard, P. E. (2018). Traffic Modeling for Aggregated Periodic IoT Data.

Hou, L., Zhao, S., Xiong, X., Zheng, K., Chatzimisios, P., Hossain, M. S., and Chen, W. (2016). Internet of Things Cloud: Architecture and Implementation. IEEE Communications Magazine, 54(11):32-39.

Ito, M. S., Antonello, R., Sadok, D., and Fernandes, S. (2014). Network level characterization of adaptive streaming over HTTP applications. In Proceedings - International Symposium on Computers and Communications. Institute of Electrical and Electronics Engineers Inc. 
Itu-r (2017). Minimum requirements related to technical performance for IMT-2020 radio interface(s) M Series Mobile, radiodetermination, amateur and related satellite services. Technical report.

ITU Recommendation G.1010 (2001). End-user multimedia QoS categories.

Jain, R. K. (1991). The Art of Computer System Performance Analysis.

Khalili, H., Papageorgiou, A., Siddiqui, S., Colman-Meixner, C., Carrozzo, G., Nejabati, R., and Simeonidou, D. (2019). Network Slicing-aware NFV Orchestration for 5G Service Platforms. Technical report.

Laghrissi, A. and Taleb, T. (2019). A Survey on the Placement of Virtual Resources and Virtual Network Functions. IEEE COMMUNICATIONS SURVEYS \& TUTORIALS, 21(2)

Laner, M., Nikaein, N., Drajić, D., Svoboda, P., Popović, M., and Krčo, S. (2014). M2M traffic and models. In Machine-to-Machine Communications: Architectures, Technology, Standards, and Applications, pages 57-86. CRC Press.

Nam, J., Seo, J., and Shin, S. (2018). Probius: Automated Approach for VNF and Service Chain Analysis in Software-Defined NFV. page 13.

Popovski, P., Trillingsgaard, K. F., Simeone, O., and Durisi, G. (2018). 5G Wireless Network Slicing for eMBB, URLLC, and mMTC: A Communication-Theoretic View. IEEE Access 6.

Rebecchi, F., Vidalenc, B., Belabed, D., Kalaboukas, K., Corsi, A., Fiorentino, G., Mladin, M., Duke, A., and Voulkidis, A. (2019). Enabling Smart Energy as a Service via 5G Mobile Network advances. Technical report, Project H2020-ICT-07-2017.

Riera, J. F., Hesselbach, X., Escalona, E., Garcia-Espin, J. A., and Grasa, E. (2014). On the complex scheduling formulation of virtual network functions over optical networks.

Rizou, S., Athanasoulis, T., Iadanza, F., Pavia, D., Breitgand, D., Weit, A., Agapiou, G., Griffin, D., Phan, K., Carrozzo, G., Moscatelli, F., Acar, U., and Meco, D. L. (2018). D3.1 - Initial Design of the 5G-MEDIA Operations and Configuration Platform. Technical report, Operations and Configurations Framework.

Rosa, R. V., Bertoldo, C., and Rothenbe, C. E. (2017). Take Your VNF to the Gym: A Testing Framework for Automated NFV Performance Benchmarking.

Rosa, R. V., Rothenberg, C. E., and Szabo, R. (2015). VBaaS: VNF Benchmark-as-aService. 2015 Fourth European Workshop on Software Defined Networks.

Sefraoui, O., Aissaoui, M., and Eleuldj, M. (2012). OpenStack: Toward an Open-Source Solution for Cloud Computing. Technical Report 03.

Sivanathan, A., Sherratt, D., Gharakheili, H. H., Radford, A., Wijenayake, C., Vishwanath, A., and Sivaraman, V. (2017). Characterizing and classifying IoT traffic in smart cities and campuses. In 2017 IEEE Conference on Computer Communications Workshops, INFOCOM WKSHPS 2017, pages 559-564. Institute of Electrical and Electronics Engineers Inc.

Taleb, T., Bagaa, M., and Ksentini, A. (2015). User mobility-aware Virtual Network Function placement for Virtual 5G Network Infrastructure, volume 2015-Septe. 